\title{
Profil Tensionnel des Patients Parkinsoniens Idiopathiques vus au Service de Neurologie de Pontarlier
}

\author{
Lemahafaka Jemissair Glorien, \\ Interne en Neurologie, Hôpital de Pontarlier, France \\ Camara Ansoumane, \\ Neurologue, Hôpital de Pontarlier, France \\ Miandrisoa Rija Mikhael, \\ Chef de Clinique en Cardiologie à la \\ Faculté de Médecine d'Antananarivo, Madagascar
}

Vallet Francis,

Praticien Hospitalier en Neurologie, Hôpital de Pontarlier, France

Tehindrazanarivelo Alain Djacoba,

Professeur en Neurologie, Faculté de Médecine,

Université d'Antananarivo, Madagascar

Doi: 10.19044/esj.2019.v15n15p133 URL:http://dx.doi.org/10.19044/esj.2019.v15n15p133

\section{Résumé}

Introduction: La maladie de Parkinson idiopathique est une affection neurodégénérative caractérisée par des troubles moteurs, l'akinésie ou bradykinésie, la rigidité, le tremblement de repos et altération des réflexes de posture voire des chutes fréquentes. Dans la pratique quotidienne, une anomalie du profil tensionnel est souvent observée chez les malades parkinsoniens. Notre objectif est de décrire le profil tensionnel pendant $24 \mathrm{~h}$ chez nos patients atteints de la maladie de Parkinson idiopathique. Méthode : Il s'agit d'une étude d'observation prospective et descriptive portant sur des patients hospitalisés de mai à octobre 2016 pour une maladie de Parkinson idiopathique au service de Neurologie à l'hôpital de Pontarlier. Nous avons inclus tous les patients ayant un score UPDRS (Unified Parkinson's Disease Rating Scale) supérieur à 12 ayant effectués un enregistrement holter TA pendant $24 \mathrm{~h}$. Résultats : Nous avons retenu 50 patients, 15 femmes et 35 hommes (soit un sex-ratio homme-femme de 2,33), âgés de 45 à 84 ans, moyenne 65,24 et ecart type 3,04. Le profil tensionnel était normal chez 10 patients soit $20 \%$. Chez 40 patients soit $80 \%$ de cas a été retrouvée une hypotension artérielle et/ou une normotension. Les pics d'apparition de l'hypotension artérielle se situaient entre $9 \mathrm{~h}$ et $15 \mathrm{~h}$. Les signes associés à cette 
hypotension artérielle étaient soit des chutes avec perte de connaissance brève chez $20 \%$, des chutes sans perte de connaissance chez $12 \%$, des vertiges chez $11 \%$, un flou visuel chez $8 \%$ et d'autres signes chez $25 \%$. Conclusion : Les manifestations cliniques particulières liées à l'hypotension artérielle méritent plus d'attention de notre part d'où l'intérêt d'une recherche systématique chez tout les malades parkinsoniens et apparentés.

Mots-clés : Maladie de Parkinson idiopathique, hypotension orthostatique

\title{
Blood Pressure Profile of Patients with Idiopathic Parkinson's Disease at the Neurology Unit of Pontarlier
}

\author{
Lemahafaka Jemissair Glorien, \\ Interne en Neurologie, Hôpital de Pontarlier, France \\ Camara Ansoumane, \\ Neurologue, Hôpital de Pontarlier, France \\ Miandrisoa Rija Mikhael, \\ Chef de Clinique en Cardiologie à la \\ Faculté de Médecine d'Antananarivo, Madagascar \\ Vallet Francis, \\ Praticien Hospitalier en Neurologie, Hôpital de Pontarlier, France \\ Tehindrazanarivelo Alain Djacoba, \\ Professeur en Neurologie, Faculté de Médecine, \\ Université d'Antananarivo, Madagascar
}

\begin{abstract}
Introduction: Idiopathic Parkinson's disease is a neurodegenerative disorder characterized by motor disorders, akinesia or bradykinesia, rigidity, resting tremor and impaired posture reflexes or even frequent falls. In daily practice, an abnormal blood pressure profile is often observed in parkinsonian patients. In this study, we described the $24 \mathrm{~h}$ blood pressure profile in our patients with idiopathic Parkinson's disease. Methodology: This is a prospective and descriptive observational study of patients hospitalized from May to October 2016 for idiopathic Parkinson's disease at the Department of Neurology at Pontarlier Hospital. We included all patients with an UPDRS (Unified Parkinson's Disease Rating Scale) score greater than 12 who made a
\end{abstract}


holter TA record for 24 hours. Results: We selected 50 patients, 15 women and 35 men (a sex ratio between men and women of 2.33), aged 45 to 84, average 65.24 and standard deviation 3.04. The blood pressure profile was normal for $24 \mathrm{~h}$ in 10 patients $(20 \%)$. The remainder, 40 patients $(80 \%)$, showed during the 24 hours of recording an alternation of arterial hypotension and normotension. Peaks of onset of arterial hypotension were $9 \mathrm{~h}$ and $15 \mathrm{~h}$. The signs associated with this low blood pressure were falls with loss of consciousness $(20 \%)$, isolated falls (12\%), vertigo (11\%), visual blur (8\%), and other signs (25\%). Conclusion: The particular clinical manifestations related to hypotension deserve more attention from us based on the interest of a systematic research in all the parkinsonian and related patients.

Keywords: Idiopathic Parkinson's disease, orthostatic hypotension

\section{Introduction}

La maladie de Parkinson est une maladie neurodégénérative par dénervation dopaminergique nigrostriatale progressive caractérisée par des symptômes moteurs et non-moteurs qui sont responsables d'une diminution de la qualité de vie et d'un retentissement important sur l'activité professionnelle (Ascherio et al., 2016). Les malades parkinsoniens vont faire face à une augmentation du risque de dépendance, notamment en raison de complications motrices (dyskinésies, fluctuations, chutes) et cognitives (déclin cognitif, hallucinations, démence) (Daniel Weintraub et al., 2008). Ainsi, la maladie de Parkinson constitue un fardeau important, aussi bien sur le plan familial que social et économique. Au cours de son évolution, la maladie de Parkinson présente des manifestations variables. Nous constatons des instabilités tensionnelles, de découverte souvent fortuite au décours le plus souvent d'une hospitalisation des patients. Ce changement de profil tensionnel constitue une comorbidité qui peut entrainer d'autres signes ou symptômes motivant souvent une hospitalisation, d'où cette étude qui a pour but de décrire le profil tensionnel des patients parkinsoniens pendant 24heures afin de mieux optimiser la prise en charge.

\section{Méthodologie}

Il s'agit d'une étude prospective et descriptive portant sur des patients hospitalisés de mai à octobre 2016 pour une maladie de Parkinson idiopathique au service de Neurologie à l'hôpital de Pontarlier, France. Nous avons inclus tous les patients diagnostiqués comme maladie de Parkinson idiopathique avec un score UPDRS (Unified Parkinson's Disease Rating Scale) supérieur à 12, ayant effectués systématiquement l'holter TA pendant $24 \mathrm{~h}$ pour dépister l'anomalie tentionnelle. Nous avons utilisé le score UPDRS dans cette étude car il recouvre toutes les situations cliniques pui peuvent être 
observées au cours de la maladie de Parkinson. Le score total est 108. La cotation est inférieur à 12/108 lorsque le malade en période de lune de miel et entre 12 à 30/108 lorsque la maladie est installée et de 30 à 80/108 chez les patients à manifestations sévères. Nous avons exclus tous les patients ayant d'autres causes d'anomalies tensionnelles (diabète, médicament, alccol, tabac), les patients remplis les critères d'inclusion mais n'ayant pas bénéficier de holter TA et/ou non interprétable en raison d'erreur technique ou ayant un holter TA avec des mesures tensionnelle insuffisante. Nous avons étudiés les paramètres socio-démographiques, la variation tensionnelle et les manifesations cliniques des anomalies tensionnelles.

\section{Résultats}

Sur 350 patients admis durant la période d'étude, nous avons 60 patients remplissent les critères d'inclusion et 10 patients sont exclus après critères d'exclusion. Nous avons finalement retenu cinquante patients (14,28\% d'admission hospitalière), 15 femmes et 35 hommes, soit un sexratio de 2,33, L'âge de nos patients allant de 45 à 84 ans dont l'âge moyen est de 65,24 ans. Parmi les patients retenus, 76\% $(\mathrm{n}=38)$ étaient au stade de complication dont le score UPDRS est entre 30 à 80 , le reste $24 \%(\mathrm{n}=12)$ au stade d'installation de la maladie avec UPDRS entre 12 à 30 . Le profil tensionnel était normal durant $24 \mathrm{~h}$ chez 10 patients $(20 \%)$. Le reste, soit 40 patients $(80 \%)$ montraient au cours des $24 \mathrm{~h}$ d'enregistrement une alternance d'hypotension artérielle et de normotension. Les pics d'apparition de l'hypotension artérielle au repos l'hypotension artériell etaient autour de 9h et 15h. Les signes associés à cette hypotension artérielle étaient soit des chutes avec perte de connaissance (20\%), des chutes isolées (12\%), des vertiges $(11 \%)$, un flou visuel $(8 \%)$ et d'autres signes (25\%) et asymptomatique (25\%).

Tableau 1. Caractéristiques de la population étudiée

\begin{tabular}{lll}
\hline Caractéristiques & Effectif $(\mathbf{n})$ & \% \\
\hline Genre & & \\
Masculin & 35 & 70 \\
Féminin & 15 & 30 \\
Age & & \\
{$[40-45]$} & 1 & 2 \\
{$[45-50]$} & 2 & 4 \\
{$[50-55]$} & 5 & 10 \\
{$[55-60]$} & 7 & 14 \\
{$[60-65]$} & 8 & 16 \\
{$[65-70]$} & 10 & 20 \\
{$[70-75]$} & 8 & 16 \\
{$[75-80]$} & 6 & 12
\end{tabular}


[80 - 85]

\section{UPDRS}

$[12-30]$

[30 - 108]

Profil TA pendant $24 \mathrm{~h}$

Normal

Avec hypotension

Anomalies associées

Chute avec perte de connaissance

Chutes

Vertiges

Flou visuel

Autres *

Asymptomatique
Autres : fatigue, malaise, nausée (sont rares et isolés)

\section{Discussion}

Cette étude a pour objet de décrire le profil tensionnel des patients parkinsoniens pendant $24 \mathrm{~h}$ au décours d'hospitalisations programmées ce qui permet de dépister les co-morbidités tensionnel de la maladie de Parkinson. Le dépistage des co-morbidités est important dans l'aide au diagnostic et de la prise en charges des patients.

Nous avons retenus 50 dossiers après critères d'inclusion et d'exclusion, soit $14,28 \%$ de toutes les admissions hospitalières durant la période d'étude. La maladie de Parkinson est une maladie fréquente, elle est la deuxième maladie neurodégénérative après la maladie Alzheimer. La maladie de Parkinson existe dans tous les pays et chez toutes les ethnies, mais avec une prévalence très variable. La prévalence de la maladie se situe entre 18 à 234 cas pour 100000 habitants dans les études en population générale, même après standardisation des résultats. Schématiquement, on peut retenir que la maladie de Parkinson est 2 à 5 fois plus fréquente dans les pays industrialises que dans les pays en voie de développement (Viallet et al., 2001) . La maladie de Parkinson est habituellement plus fréquente chez les hommes que chez les femmes, avec un sex-ratio d'environ 1,5 homme pour une femme. Elle pourrait s'expliquer par certaines expositions professionnelles plus fréquentes chez les hommes, à l'effet neuroprotecteur d'œstrogènes ou à un facteur génétique (Bourdaud Laure, 2013; De Rijk et al., 2000).

L'âge des patients se situe entre 45 et 84ans. L'âge moyen est de 65 ans. Il est évident que la maladie de Parkinson touche souvent les sujets âgés et sa prévalence augmente avec l'âge. Selon la meta-analyse Europarkinson reprenant toutes ces études, en Europe cette pathologie atteindrait 1,7 \% de la 
population après 65 ans, allant de $0,6 \%$ entre 65 et 69 ans à 2,6 \% entre 85 et 89 ans (De Rijk et al., 2000; Nicolas et al., 2015).

L'anomalie tensionnelle caractérisée par l'alternance de l'hypotension et de la normotension est retrouvée chez $80 \%$ de nos patients. La maladie de Parkinson est une maladie chronique évolutive. Le mécanisme de la survenue d'hypotension artérielle n'a pas encore bien identifié mais plusieurs hypothèses ont été avancées. L'hypotension artérielle que ce soit au repos et/ou en orthostatisme est assez fréquente avec une prévalence de près de 60 $\%$ dans la maladie de Parkinson, corrélée, quand elle est symptomatique, avec la durée d'évolutio de la maladie en rapport avec la sévérité de la maladie, les doses de levodopa et d'agoniste dopaminergique. Cette prévalence augmente en cas de préexistence d'une hypertension artérielle systolique.

Sur le plan physiopathologique, elle est due à une dysfonction sympathique cardiaque et vasomotrice alors que le reflexe barorécepteur est peu altéré. La prédominance des lésions du système sympathique en pré- ou post ganglionnaire n'est pas clairement établie. L'hypotension orthostatique est définie par la perte, lors des 3 premières minutes (mais souvent plus tardive dans la maladie de Parkinson), du passage actif ou passif en orthostatisme de $20 \mathrm{~mm} \mathrm{Hg}$ de pression artérielle systolique ou de $10 \mathrm{~mm} \mathrm{Hg}$ de pression diastolique, éventuellement associée à des symptômes et corrigée par le retour en clinostatisme, s'accompagnant ou non d'une accélération du rythme cardiaque. Elle peut exister spontanément et sera, à un stade plus évolué, aggravée par les traitements antiparkinsoniens du fait de leurs effets vasodilatateur et sympathicolytique, justifiant une adaptation des doses si l'hypotension est symptomatique. L'hypotension orthostatique voit sa prévalence augmenter avec l'âge et avec la préexistence d'une hypertension artérielle systolique ; elle est fréquemment accentuée en période postprandiale (Oka et al., 2007; Zesiewicz et al., 2010 ; Dewey, 2004).

Ces anomalies tensionnelles pourraient être asymptomatiques mais nous pouvons trouvés des symptômes en rapport avec l'hypotension orthostatique et/ou syndrome de bas débit cérébrale.

Selon la littérature, l'hypotension est tantôt asymptomatique, tantôt responsable (dans 20 à $25 \%$ des cas), lors des changements de position trop rapides ou en période postprandiale, de lipothymies, de troubles visuels, d'une sensation d'asthénie, de sensations vertigineuses, d'une instabilité posturale, de chutes sans perte de connaissance ou plus rarement de syncopes (Zesiewicz et al., 2010 ; Azulay et al., 2008).

\section{Conclusion}

Au total, la maladie de Parkinson est une maladie neurodégénerative chronique évolutive avec des symptômes moteurs et non moteurs. L'anomalie tensionnelle est fréquente au cours de la maladie de Parkinson. Les 
manifestations cliniques particulières liées à l'hypotension artérielle même en dehors de l'orthostatisme méritent plus d'attention de notre part car elles majorent la difficulté de la prise en charge des malades, et devrait être recherchée systématiquement par Holter TA qui pourrait être un bilan de routine lors de la prise en charge de la maladie de Parkinson.

\section{References:}

1. Ascherio, A. \& Schwarzschild, MA. (2016). The epidemiology of Parkinson's disease: risk factors and prevention; Lancet Neurol; 15(12):1257-1272. doi: 10.1016/S1474-4422(16)30230-7

2. Azulay, JP., Durif, F. \& Rogez, R. (2008). Precoce survey: a new selfassessment patient card for early detection and management of Parkinson disease fluctuations. Rev Neurol (Paris); 164 (4) : 354-62.

3. Daniel, W., Cynthia, L., Comella, \& Stacy Horm (2008). Parkinson's Disease: physiopathology, symptoms, burden, diagnosis and assessment: Am J Manag Care; 14: S40 - S48

4. Dewey, RB. (2004). Autonomic dysfunctions in Parkinson's disease. NeurolClin; 22: S127-39.

5. De Rijk, MC., Launer, LJ., \& Berger, K. (2000). Prevalence of Parkinson's disease in Europe : a collaborative study of populationbased cohorts. Neurology ; 54 (Sup.5) : $31-3$.

6. Ferreira, J., Galitzky, M., Montastruc, JL., \& Rascol, O. (2000). Sleep attacks and Parkinson's disease treatment. Lancet; 355: 1333-4.

7. Krystkowiak, P., Tison, F., \& Bonnet, AM. (2006). Echelles et questionnaires d'évaluation de la maladie de Parkinson ; Encyclopédie Médico-chirurgicale neurologie ; Elsevier Masson, 17 - 035- A - 83.

8. Nicolas Carrière \& Luc Defebvre (2015). Syndrome parkinsonien à début précoce : Quel bilan, à quel moment ? Neurologies (18) 176; 76 -84 .

9. Oka, H., Yoshioka, M. \& Onouchi, K. (2007). Characteristics of orthostatic hypotension in Parkinson's disease. Brain ; 130 (9) : 242532.

10. Viallet, F., Gayraud, D., Bonnefoi, B., Kyriacou, et al. (2001). Aspects cliniques et thérapeutiques de la maladie de Parkinson ; Encyclopédie Médico-chirurgicale, Elsevier Masson ; 17 - 060 - A - 50

11. Zesiewicz, TA., Sullivan, KL., \& Arnulf, I. (2010). Practice Parameter: treatment of nonmotor symptoms of Parkinson disease: report of the Quality Standards Subcommittee of the American Academy of Neurology. Neurology; 74 (11): 924-31. 